\section{A computer program to compute Scheffë's homogeneity of variance statistic and estimate its power}

\author{
GERARD J. SMITS \\ Boston University, Boston, Massachusetts 02215
}

Many statistical tests assume homogeneity of variance, and may be affected by the violation of this assumption. This is particularly the case with analysis of variance for unequal cell size. For this reason, a powerful and robust test to detect heterogeneity of variance is desirable. Unfortunately, most commonly used tests of homogeneity of variance are sensitive to departures from normality, especially with respect to departures from nonzero kurtosis (Box, 1953). A test that has been shown to be robust to violation of distribution assumptions is the Scheffe test, described by Winer (1971, pp. 219.220).

In the Scheffe test, each treatment group is randomly divided into a number of subsamples, and their variances are computed. The logarithms of the variances are taken, and an analysis of variance is performed.

Two major drawbacks exist with the Scheffe procedure. First, because the grouping of subsamples is random, the test does not yield a unique solution. This has led some to reject it as unacceptable (Brown \& Forsythe, 1974). Second, the test generally has poor power to detect existing heterogeneity of variance.

Description. The present program uses a Monte Carlo sampling procedure to minimize the uniqueness problem and to provide information on the power to detect a specified degree of heterogeneity of variance. Essentially, the program computes the F statistic for a randomly generated group of subsamples and repeats this process a specified number of times, each time operating on a newly generated set of subsamples. The size of the loop is presently set at 100 . In this manner, a mean $F$ ratio, based on the series of replications, provides a stable estimate of the $F$ ratio, with reduced influence from random subsample formation. To provide information on power to detect a given level of heterogeneity of variance, a series of runs can also be performed on a set of internally generated normal random deviates with a built-in degree of variance heterogeneity. In this way, a simulation, conforming to a researcher's design in terms of sample size and number of treatment groups, yields an empirical estimate of power to detect a degree of heterogeneity of variance. The number of replications performed to provide an estimate of power is currently set at 500 .

Input. To run the basic program, two cards must be specified. One defines the number of treatment groups in the design, and the other sets the Ns for each of the treatment groups. If a test of power is desired, two additional cards are specified. The first defines the degree of variance magnification that the researcher wishes to detect; the second is the F ratio that must be reached to obtain a given significance level. The input format for the data is presently set so that the data from Treatment Group 1 are on the first card; from Group 2 they are on the second card, and so on. An F1.0 format is used but this may be readily changed. No formatting is necessary for the test of power, as the data are generated within the program. The size of subgroups within each treatment group is handled within the program to optimize the power of the test. This may be changed if different subsample sizes are desired.

Output. A mean $\mathrm{F}$ ratio is printed with its associated degrees of freedom. The standard error of the $F$ ratio is given with $95 \%$ and $99 \%$ confidence intervals of the estimate. If a test of power is performed, the power to detect the specified heterogeneity of variance is given along with the standard error of the estimate.

Computer and Language. The program was written in FORTRAN IV and was tested on an IBM 370/158. The program requires approximately 9.5 bytes of core, and it takes $8 \mathrm{sec}$ to compile and run. An additional $60 \mathrm{sec}$ must be added if a test of power is to be performed. These estimates are based on a run with five treatment groups, each with Ns of 80 .

Availability. A fully documented listing of the program can be obtained free of charge from Gerard J. Smits, Department of Psychology, Boston University, Boston, Massachusetts 02215 .

\section{REFERENCES}

Box, G. E. P. Non-normality and tests on variances. Psychometrika, 1953, 40, 318-335.

Brown, M. B., \& Forsythe, A. B. Robust tests for the equality of variances. Journal of the American Statistical Association, 1974, 69, 364-367.

WINER, B. J. Statistical principles in experimental design. (2nd ed.). New York: McGraw-Hill, 1971.

(Accepted for publication December 28, 1978.) 